\title{
A Novel Decentralized Responsibilizing Primary Frequency Control
}

\author{
M. H. Syed, E. Guillo-Sansano, S. M. Blair, A. J. Roscoe, and G. M. Burt
}

\begin{abstract}
There is an emerging need to prioritize remedial frequency control measures closer to the source of an imbalance event. This approach, referred to herein as responsibilization, is challenging to achieve within the conventional operating times of Primary Frequency Control (PFC) and therefore novel methods are sought. In this letter, a novel decentralized PFC is proposed which relies on transient phase offset to achieve fast responsibilization autonomously. The effectiveness of the proposed control is demonstrated by real-time simulations and its stability assessed by small-signal analysis. This development will lead to increased system resilience during imbalance events.
\end{abstract}

Index Terms-Adaptive droop, decentralized control, primary frequency control and small-signal stability.

\section{INTRODUCTION}

$\mathrm{P}$ RIMARY Frequency Control (PFC) in synchronous power systems is designed such that each Load Frequency Control (LFC) area responds proportionally to their capacity based on the frequency droop slope set by the system operator [1], [2]. An imbalance event is caused by loss of generation or load, and is referred to as 'event' henceforth. The need to prioritize PFC response closer to the source of event to ensure stable operation in a future changing grid has been demonstrated in [3] and further discussed in [4]. The term responsibilization refers to the prioritization of remedial measures closer to the origin of an event, driving towards a new paradigm of increased decentralization. Although responsibilization is desired, PFC's operation at a much faster timescale than Secondary Frequency Control (SFC), where responsibilization is conventionally in place, presents a challenge and there have been no satisfactory decentralized alternatives available. A pragmatic approach to responsibilization is to enforce the LFC with an event (henceforth referred to as 'local area') to contribute more PFC response to the event.

Responsibilization could be achieved by means of adapting the droop slopes of the LFC areas in real-time, i.e., allocation of a higher active-power response contribution (lower frequency droop percentage) to the local area. This can be done if the location of the event can be detected within the time scale of PFC. A number of works discuss adaptive droops for microgrids [5], [6], but only [7] for PFC of LFC areas. In [7], responsibilizing PFC for LFC areas is introduced, where the droop slope of the area is adapted by means of event detection, but the detection is centralized within the LFC area, subject to measurement uncertainty and relies extensively on communication infrastructure.

This letter proposes an alternative novel decentralized PFC, where responsibilization is achieved by means of measuring the transient phase offset (TPO) within each of the LFC areas. The proposed approach is fully decentralized as it relies on

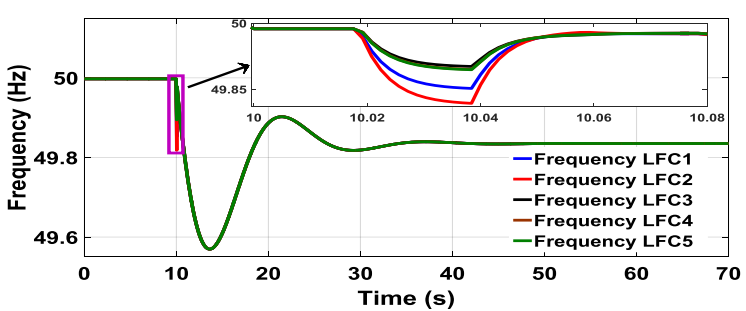

(a)

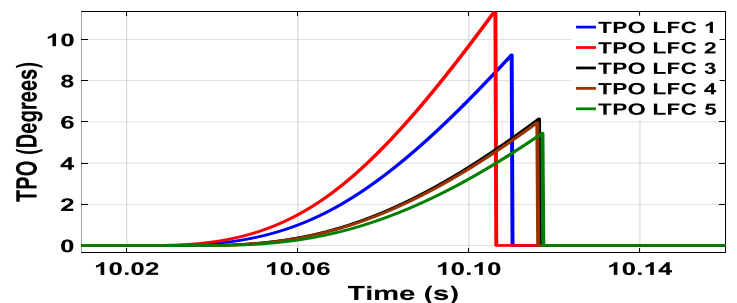

Fig. 1. Reference event: (a) frequency and (b) TPO

local measurement only and requires no form of communication. The performance of the proposed control is verified by real-time simulations and corroborated by smallsignal analysis.

\section{RESPONSIBILIZING PRIMARY FREQUENCY CONTROL}

In this section, the TPO based method of responsibilization is presented.

\section{A. Fast Event Location Detection by Transient Phase Offset}

Any sudden imbalance between generator mechanical power and load leads to a perceived change in frequency, in high or low inertia systems, due to the changing phase angles across network impedances, as active power flows change. The local Rate of Change of Frequency (RoCoF) in response at time instant $k$ can be estimated as:

$$
\text { RoCoF }=\left(f_{k}-f_{k-T}\right) / T H z / s
$$

where $T$ represents the measurement window length. Essentially, $R o C o F$ is an estimate of local $\left(d^{2} \emptyset / d t^{2}\right) / 360$ over the window $T$, for phase angle $\varnothing$. The TPO of a system relative to a stable frequency can then be estimated as $[8$, eq. (6.2)]:

$$
\emptyset_{o}=360 \int\left(\int R o C o F \cdot d t\right) \cdot d t, \emptyset \text { in degrees }
$$

Equation (2) estimates local deviations of phase, from a linear phase ramp extrapolated from pre-event conditions. The preevent non-zero values of phase and frequency are removed via the double differentiation, allowing local phase deviation to be reconstructed via double integration.

Upon occurrence of an event, the TPO is larger when measured geographically closer to the event than further away. Therefore, in a synchronous power system that is divided into a number of LFC areas, a local TPO measurement can be utilized to quickly and autonomously detect if an area should contribute more to PFC than other areas. To illustrate this 


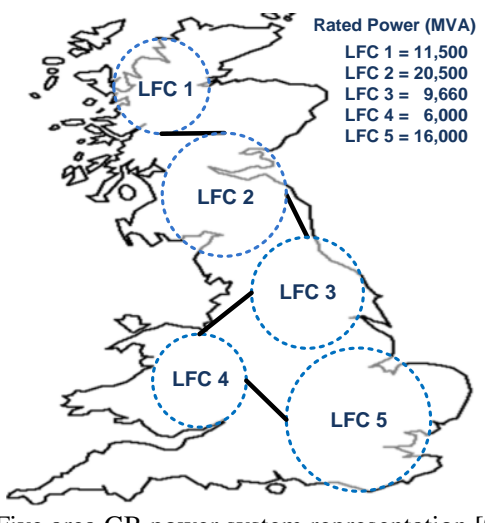

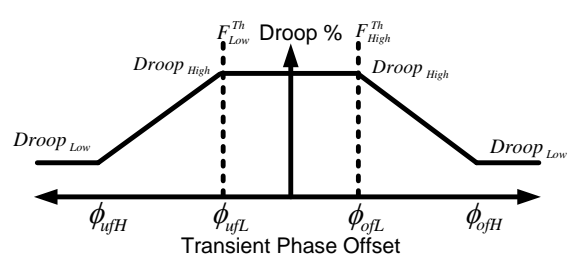

(b) Proposed PFC

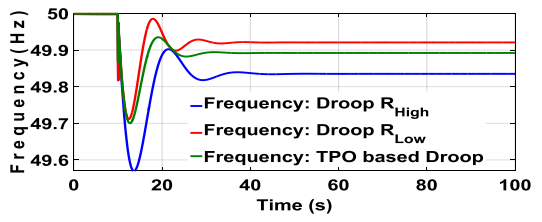

(c) Frequency response comparison

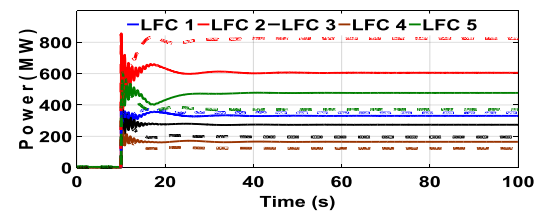

(d) LFC power output comparison

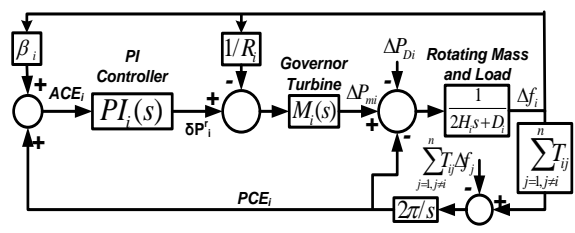

(e) n-area frequency response model

(a) Five area GB power system representation [9]

Fig. 2. Power system representation, proposed droop curve, system response to $1850 \mathrm{MW}$ generation loss and n-area frequency response model.

further, consider the response of a five LFC area reduced dynamic model of the Great Britain (GB) power system, to an 1850 MW loss of generation event in LFC 2 presented in Fig. 1 (a). The model parameters can be found in [9]. One frequency measurement is taken in each of the LFC areas, and Fig. 1 (b) shows that the observed TPO is the largest in LFC 2. In a similar manner, the next largest observed TPO is for LFC 1 that is next closest to the event (Fig. 2 (a)). TPO is much more immune to any measurement noise than RoCoF [10].

\section{B. TPO based Responsibilization}

The droop curve for the proposed control is presented in Fig. 2 (b) and is designed as follows: (i) the lower and higher frequency thresholds beyond which the droop is adaptive are defined as $F_{\text {Low }}^{T h}$ and $F_{H i g h}^{T h}$ respectively, (ii) the lower and the higher droop percentages are defined as $R_{\text {Low }}$ and $R_{\text {High }}$ respectively, (iii) the smallest and largest event size accommodated within this design are defined as $P_{\text {Low }}$ and $P_{H i g h}$ respectively, and (iv) the TPO thresholds ( $\emptyset_{U f}^{T h}$ for under frequency events and $\emptyset_{\text {of }}^{T h}$ for over frequency events) are then determined as:

$\emptyset_{U f}^{T h}=\left\{\begin{array}{l}\emptyset_{u f L}=\frac{\sum_{i=1}^{n} \phi_{o}^{i}}{n}, P_{L o w}^{+} \\ \emptyset_{u f H}=\frac{\sum_{i=1}^{n} \phi_{o}^{i}}{n}, P_{H i g h}^{+}\end{array} \quad \emptyset_{O f}^{T h}=\left\{\begin{array}{l}\emptyset_{o f L}=\frac{\sum_{i=1}^{n} \phi_{o}^{i}}{n}, P_{\text {Low }}^{-} \\ \emptyset_{o f H}=\frac{\sum_{i=1}^{n} \phi_{o}^{i}}{n}, P_{H i g h}^{-}\end{array}\right.\right.$

where $n$ is the total number of LFC areas, $\emptyset_{0}^{i}$ is the TPO observed in LFC area $i$ with an event of defined size. $P^{+}$ indicates an increase in net load and $P^{-}$a decrease. Therefore, the TPO is continuously monitored within all the LFC areas and upon occurrence of an event that causes a deviation in frequency beyond $F_{\text {Low }}^{T h}$ or $F_{\text {High }}^{T h}$, the droop value based on the observed TPO is utilized. This value of droop is latched until the frequency of the system is restored within the error margin $(\varepsilon)$ defined. An increase in droop percentage corresponds to a decrease in response. It should be noted that the values of droop are a design choice, and the applicability of the proposed approach remains the same for any value $R_{\text {High }}$ and $R_{\text {Low }}$ satisfying: $R_{\text {High }}, R_{\text {low }} \in \mathbb{R}_{>0}, \forall R_{\text {High }}>R_{\text {Low }}$.

\section{Performance Evaluation}

A study is presented for the five area GB power system. The control parameters have been chosen as $T=0.02 \mathrm{~s}$, $F_{\text {Low }}^{\text {Th }}=49.8 \mathrm{~Hz}, F_{\text {High }}^{\text {Th }}=50.2 \mathrm{~Hz}, R_{\text {Low }}=5 \%, R_{\text {High }}=12 \%$, $P_{\text {Low }}=500 \mathrm{MW}$, and $P_{\text {High }}=1850 \mathrm{MW}$. As per the design procedure, the TPO thresholds are obtained as $\emptyset_{u f L}=2$, $\emptyset_{u f H}=12, \emptyset_{o f L}=2$ and $\emptyset_{o f H}=12$. The performance of the proposed control has been analyzed by means of simulations and small-signal stability analysis subject to a generation loss of $1850 \mathrm{MW}$ in LFC 2.

\section{A. Simulations}

System frequency response is shown in Fig. 2 (c) to be stable and between fixed droop response of $R_{\text {Low }}$ and $R_{\text {High }}$. The PFC power contribution of each LFC is presented in Fig. 2 (d). The solid line represents the system response with fixed droop and the dotted line represents system response with the proposed control. LFC 2 increases its contribution to the event, demonstrating greater responsibilization.

\section{B. Small Signal Analysis}

It is essential to assess the system stability with both PFC and SFC. The system frequency response model of an $n$-area interconnected power system is presented in Fig. 2 (e). The state-space model of n-area system can be represented as [11]:

$$
\left\{\begin{array}{c}
\dot{x}=A x+B u+F w \\
y=C x
\end{array}\right.
$$

where the state vector $x=\left[x_{1}, x_{2}, x_{3}, x_{4}\right]^{T}$, the control vector $u=\left[\delta P_{1}^{r}, \ldots, \delta P_{n}^{r}\right]^{T}$, and the disturbance vector $w=$ $\left[\Delta P_{D 1}, \ldots, \Delta P_{D n}\right]^{T}$. The internal states can be represented as $x_{1}=\left[\Delta f_{1}, \ldots, \Delta f_{n}\right], x_{2}=\left[\Delta P_{m 1}, \ldots, \Delta P_{m n}\right], x_{3}=\left[P C E_{1}, \ldots, P C E_{n}\right]$, and $x_{4}=\left[\int A C E_{1}, \ldots, \int A C E_{n}\right] . A C E_{i}$ is the area control error, $P C E_{i}$ is the power control error, $\delta P_{i}^{r}$ is the regulation power output from the PI controller, $\Delta P_{m i}$ is the governor power output, and $\Delta P_{D i}$ is the power disturbance within LFC area $i$. The coefficient matrices $A, B$, and $C$ can be found in [11]. Based on the model presented above, small-signal analysis has been conducted on the five area power system with the aforementioned disturbance. For the given disturbance, the droop value of local area is decreased in steps of $0.5 \%$ and for each step reduction the non-event LFC droop values are changed by parametric sweep in steps of $0.5 \%$. For all the cases, all the non-zero eigenvalues lie on the left half plane, demonstrating stable operation for the chosen design parameters.

\section{CONCLUSIONS}

In this letter, a novel decentralized PFC is proposed. The control achieves fast and autonomous responsibilization by means of local TPO observation within the LFC areas. The stability of the proposed control is assessed by means of 
small-signal analysis. The potential advantages of the proposed control are: (i) the prioritization of local response to a local imbalance, reducing the divergence from planned system conditions and hence minimizing the operational implications of the disturbance, and (ii) supporting enhanced scalability in the future grid given the autonomy of the approach.

\section{REFERENCES}

[1] "Policy P1: Load-Frequency Control and Performance", in Continental Europe Operation Handbook, ENTSO-E, 2016.

[2] European Commission. Commission Regulation (EU) 2017/1485 of 2 August 2017 establishing a guideline on electricity transmission system operation. [Online]. Available: http://eur-lex.europa.eu/legalcontent/EN/TXT/?uri=CELEX:32017R1485

[3] V. Terzija, "Adaptive underfrequency load shedding based om magnitude of disturbance estimation", IEEE Trans. Power Syst., 2006.
[4] D. Wilson, et al,. "Advances in wide area monitoring and control to address emerging requirements related to inertia, stability and power transfer in the GB power system", CIGRE, 2016.

[5] Y. Han, et al., "Review of Active and Reactive Power Sharing Strategies in Hierarchical Controlled Microgrids", IEEE Trans. Power Electron., 2017.

[6] S. M. Malik, et al., "Voltage and frequency control strategies of hybrid AC/DC microgrid: a review", IET GTD, 2017.

[7] E. Rikos, et al., "Adaptive Fuzzy Control for Power-Frequency Characteristic Regulation in High-RES Power Systems", Energies, 2017.

[8] A. J. Roscoe, "Measurement, control and protection of microgrids at low frame rates supporting security of supply", Ch. 6, PhD thesis, University of Strathclyde, 2009.

[9] A. Emhemed, et al., "Studies of dynamic interactions in hybrid ac-dc grid under different fault conditions using real time digital simulation", IET Int. conf. on AC and DC Power Transmission, 2017.

[10] F. Ding, et al., "Peak-ratio analysis method for enhancement of LOM protection using M class PMUs", IEEE Trans. Smart Grid, 2016.

[11] N. V. Ramana, "Two-Area Load Frequency Control", in Power System Operation and Control, Pearson Education India, Ch. 6, pp. 115-131. 